No. 626

March 2020

Strong Stationarity for Optimal Control of Variational Inequalities of the Second Kind

C. Christof, C. Meyer, B. Schweizer, S. Turek 


\title{
Strong Stationarity for Optimal Control of Vari- ational Inequalities of the Second Kind
}

\author{
Constantin Christof, Christian Meyer, Ben Schweizer and Stefan \\ Turek
}

\begin{abstract}
This paper is concerned with necessary optimality conditions for optimal control problems governed by variational inequalities of the second kind. So-called strong stationarity conditions are derived in an abstract framework. Strong stationarity conditions are regarded as the most rigorous ones, since they imply all other types of stationarity concepts and are equivalent to purely primal optimality conditions. The abstract framework is afterwards applied to four application-driven examples.
\end{abstract}

Mathematics Subject Classification (2000). 49K24 35J85 49J40 90C31 49J27.

Keywords. Optimal Control of Variational Inequalities, Sensitivity Analysis, Strong Stationarity.

\section{Introduction}

This article is concerned with optimal control problems governed by variational inequalities (VIs) of the second kind. Optimal control problems of this type arise in various applications, for instance in the optimization of elastoplastic deformation processes, type-II-semiconductors or rheological fluids, see [7, 13, 29].

Optimal control problems governed by VIs provide the particular challenge that the control-to-state mapping, i.e., the solution mapping of the VI under consideration, is frequently not Gâteaux-differentiable. Therefore, the standard adjoint approach for the derivation of Karush-Kuhn-Tucker (KKT) conditions that is widely used in optimal control is not applicable when it comes to optimization problems constrained by VIs. For this reason, the derivation of qualified optimality systems involving dual variables is all but elementary in the context of optimal control of VIs. There are multiple strategies to overcome this issue, among them various smoothing and exact penalization techniques. We only refer to [8] and the various references therein for a broad overview. All these approaches yield 
necessary optimality conditions of different strength, see [15] for a survey of the multiple stationarity concepts. The most rigorous notion of stationarity is called strong stationarity. The characteristic feature of a system of strong stationarity is that it implies all the other stationarity conditions and is moreover equivalent to a purely primal optimality condition, called Bouligand(B)-stationarity.

To the best of our knowledge, there are in principle two ways to establish strong stationarity conditions for optimal control problems governed by VIs. Both approaches presume that the control-to-state map is at least directionally differentiable. The first approach was initiated by [19] and is to some extent based on the idea to take the "linearized" states as variations and not feasible controls as usually done in the derivation of first-order conditions. The alternative approach for the derivation of strong stationarity conditions originates from finite dimensional programs with complementarity constraints and works as follows: First one "linearizes" the optimal control problem by means of the directional derivative of $S$. Then, one defines auxiliary problems by fixing variables in the "linearized" problem. The latter are standard optimal control problems, which, under suitable assumptions, allow the derivation of KKT conditions. The latter then imply the desired strong stationarity conditions. For details on this approach, we refer to $[14,26,27]$.

Here, we follow the first approach of [19] and generalize it for the optimal control of VIs of the second kind. Mignot's approach has mostly been applied to optimal control of VIs of the first kind, see, e.g., [1, 16, 20]. However, it turns out that the method of proof is essentially based on a particular structure of the directional derivative of the control-to-state mapping, which is also frequently observed in case of VIs of the second kind. Therefore, by slightly generalizing Mignot's approach, we construct a general framework for the derivation of strong stationarity conditions. We then apply this general result to four application driven problems. First we show that the obstacle problem fits into our general framework, which allows us to deduce the classical results by Mignot. As a second example, we consider the optimal control of static elastoplasticity in primal formulation, which is a VI of the second kind. As in case of the obstacle problem, the control-to-state map of the VI of static elastoplasticity provides a directional derivative with the desired structure without any further assumptions. This differs from our last two examples, which cover VIs of the second kind in the Sobolev space $H^{1}(\Omega)$ involving $L^{1}$-norms. Here, we need additional assumptions that ensure the existence of directional derivatives with certain properties in order to apply our general framework. In case of the so-called (generalized) lasso problem, these assumptions can be directly verified once a solution of the VI is given. In contrast to this, in our last example, which stems from an application in rheological fluid mechanics, these assumptions are of rather intrinsic nature and may be hard to verify in practice.

It should be mentioned that this article is based on the PhD-thesis of Constantin Christof, which was written within project P16 of the DFG priority program 1962. 


\section{Notation}

The dual of a linear normed space $X$ is denoted by $X^{*}$. If $x \in X$ and $g \in X^{*}$, we write for the dual pairing $g(x)=\langle g, x\rangle_{X}$. In case that the context is clear, we sometimes neglect the index and simply write $\langle\cdot, \cdot\rangle$ for a dual pairing. If $X$ is a Hilbert space, we denote the corresponding scalar product by $(\cdot, \cdot)_{X}$. The space of linear and bounded operators from $X$ to another linear normed space $Y$ is denoted by $\mathcal{L}(X, Y)$. If $X$ is continuously embedded in $Y$ in the sense of [22, Definition 4.19], then we write $X \hookrightarrow Y$. If this embedding is dense, we write $X \hookrightarrow^{d} Y$.

\section{Strong Stationarity in an Abstract Framework}

Throughout this section, we consider the following abstract optimal control problem:

$$
\left.\begin{array}{cl}
\min & J(y, u) \\
\text { s.t. } & (y, u) \in Y \times U, \\
& y=S(u), \quad u \in U_{\mathrm{ad} .}
\end{array}\right\}
$$

On the data in $(\mathrm{P})$, we impose the following

Assumption 2.1 (Standing Assumptions). For the data in $(\mathrm{P})$, we assume the following:

- $U$ is a Hilbert space,

- $Y$ is a linear normed space,

- the objective $J: Y \times U \rightarrow \mathbb{R}$ is Fréchet-differentiable,

- $S: U \rightarrow Y$ is a continuous mapping,

- the set of admissible controls $U_{\mathrm{ad}} \subset U$ is non-empty, closed, and convex.

For the rest of this section, we tacitly assume that the above assumption is fulfilled without mentioning it every time. Moreover, in all what follows, let $(\bar{y}, \bar{u}) \in Y \times U_{\text {ad }}$ with $\bar{y}=S(\bar{u})$ be an arbitrary, but fixed local minimizer of $(\mathrm{P})$.

As indicated in the introduction, we are interested in the derivation of necessary optimality conditions. The particular challenge in case of $(\mathrm{P})$ is that we do not assume $S$ to be Gâteaux-differentiable. Therefore, standard techniques cannot be applied to establish an optimality condition involving dual variables. In contrast to this, purely primal optimality conditions can be derived by classical arguments:

Proposition 2.2 (Bouligand Stationarity). Suppose that $S$ is directionally differentiable at $\bar{u}$. Then there holds

$$
\left\langle\partial_{y} J(\bar{y}, \bar{u}), S^{\prime}(\bar{u} ; h)\right\rangle_{Y}+\left\langle\partial_{u} J(\bar{y}, \bar{u}), h\right\rangle_{U} \geq 0 \quad \forall h \in \operatorname{cone}\left(U_{\mathrm{ad}}-\bar{u}\right),
$$

where cone $\left(U_{\mathrm{ad}}-\bar{u}\right):=\left\{\alpha(u-\bar{u}): u \in U_{\mathrm{ad}}, \alpha>0\right\}$ denotes the conic hull of $U_{\text {ad }}-\bar{u}$.

Proof. Since $S$ is directionally differentiable and $J$ is Fréchet-differentiable and thus Hadamard directionally differentiable, we can apply the chain rule, which immediately gives the assertion (see [2, Prop. 2.47]). 
Throughout this article, the condition in (2.1) is termed B-stationarity and, accordingly, a point $\bar{u} \in U_{\text {ad }}$ fulfilling this condition is called B-stationary.

As indicated above, our aim is to deduce an optimality system containing dual variables from (2.1). Since $h \mapsto S^{\prime}(\bar{u} ; h)$ is in general not linear, the standard adjoint calculus cannot be applied from the shelf. In order to cope with this challenge, we need the following additional assumption on the structure of the directional derivative of $S$ at $\bar{u}$ :

Assumption 2.3 (Directional Differentiability of the Control-to-State Map). The map $S$ is directionally differentiable in $\bar{u}$ in every direction $h \in \operatorname{cone}\left(U_{\mathrm{ad}}-\bar{u}\right)$ and its directional derivative $\delta=S^{\prime}(\bar{u} ; h)$ in direction $h \in \operatorname{cone}\left(U_{\mathrm{ad}}-\bar{u}\right)$ is characterized as the solution of the following VI of the first kind:

$$
\delta \in \mathcal{K}(\bar{y}), \quad\langle A(\bar{y}) \delta, v-\delta\rangle_{V_{\bar{y}}} \geq(h, v-\delta)_{U} \quad \forall v \in \mathcal{K}(\bar{y})
$$

where

- $V_{\bar{y}}$ is a Hilbert space such that $V_{\bar{y}} \hookrightarrow Y$ and $V_{\bar{y}} \hookrightarrow^{d} U$,

- $A(\bar{y}) \in \mathcal{L}\left(V_{\bar{y}}, V_{\bar{y}}^{*}\right)$ is a strongly monotone operator,

- $\mathcal{K}(\bar{y}) \subset V_{\bar{y}}$ is a non-empty, closed, and convex cone.

As indicated by the subscript, $V_{\bar{y}}$ as well as $A(\bar{y})$ and $\mathcal{K}(\bar{y})$ may well depend on $\bar{y}=S(\bar{u})$.

In the following, we will identify $U$ with its dual (by the Riesz theorem), which gives rise to the Gelfand triple:

$$
V_{\bar{y}} \hookrightarrow U \cong U^{*} \hookrightarrow V_{\bar{y}}^{*}
$$

In this spirit, we will frequently interpret elements in $V_{\bar{y}}$ as elements in $U$ without mentioning the respective embedding operator. Similarly, we neglect the embedding operator, when $U \cong U^{*}$ is treated as a subset of $V_{\bar{y}}^{*}$. In addition, an element $g$ of $Y^{*}$ is considered as an element of $V_{\bar{y}}^{*}$ via $E^{*} g$, where $E \in \mathcal{L}\left(V_{\bar{y}}, Y\right)$ is the embedding operator from Assumption 2.3. For ease of notation, we will also omit $E^{*}$ in the following.

Unfortunately, strong stationarity conditions are in general not necessary for local optimality, as the counterexamples in $[18,25]$ show. Therefore, additional conditions are required and, in our case, we rely on the following

Assumption 2.4 (Critical Constraint Qualification). The conic hull cone $\left(U_{\mathrm{ad}}-\bar{u}\right)$ is dense in $U$, i.e., $\overline{\operatorname{cone}\left(U_{\mathrm{ad}}-\bar{u}\right)}=U$.

Remark 2.5. Assumption 2.4 is rather restrictive. For instance, it does in general not allow us to consider classical box constraints for the control, unless additional assumptions are fulfilled, which cannot be checked a priori (as usual for constraint qualifications), see, e.g., [25]. Note that, in case of box constraints, Assumption 2.4 is fulfilled, if $\bar{u}$ does not touch the bounds a.e. in the domain.

Since $A(\bar{y})$ is strongly monotone, the VI in (2.2) does not only possess a solution for every right-hand side in $\operatorname{cone}\left(U_{\mathrm{ad}}-\bar{u}\right)$, but also for inhomogeneities 
in $V_{\bar{y}}^{*}$. We denote the associated solution operator by $G_{\bar{y}}: V_{\bar{y}}^{*} \rightarrow V_{\bar{y}}$ so that $\left.G_{\bar{y}}\right|_{\text {cone }\left(U_{\text {ad }}-\bar{u}\right)}(\cdot)=S^{\prime}(\bar{u} ; \cdot)$. Due to the strong monotonicity of $A(\bar{y})$, this solution operator is globally Lipschitz continuous, i.e.,

$$
\left\|G_{\bar{y}}(g)-G_{\bar{y}}(h)\right\|_{V_{\bar{y}}} \leq L\|g-h\|_{V_{\bar{y}}^{*}} \quad \forall g, h \in V_{\bar{y}}^{*}
$$

with a Lipschitz constant $L>0$, whose potential dependency on $\bar{y}$ is suppressed for ease of notation.

As indicated above, we cannot define an adjoint state by means of the adjoint operator associated with the derivative of the control-to-state map, since the latter is nonlinear w.r.t. the direction. Instead, we introduce an adjoint state by extending the partial derivative $\partial_{u} J(\bar{y}, \bar{u})$ to the dual of $V_{\bar{y}}$, which implies that the gradient equation coupling adjoint state and optimal control is automatically fulfilled.

Lemma 2.6. There exists $p \in V_{\bar{y}}$ such that

$$
(p, h)_{U}+\left\langle\partial_{u} J(\bar{y}, \bar{u}), h\right\rangle_{U}=0 \quad \forall h \in U .
$$

Moreover, for all $h \in V_{\bar{y}}^{*}$, it holds

$$
\left\langle\partial_{y} J(\bar{y}, \bar{u}), G_{\bar{y}}(h)\right\rangle_{V_{\bar{y}}}-\langle h, p\rangle_{V_{\bar{y}}} \geq 0 .
$$

Proof. From (2.1), we know that

$$
\left\langle\partial_{y} J(\bar{y}, \bar{u}), G_{\bar{y}}(h)\right\rangle_{V_{\bar{y}}}+\left\langle\partial_{u} J(\bar{y}, \bar{u}), h\right\rangle_{U} \geq 0 \quad \forall h \in \operatorname{cone}\left(U_{\mathrm{ad}}-\bar{u}\right)
$$

and, consequently, the global Lipschitz continuity of $G_{\bar{y}}$ implies in view of $G_{\bar{y}}(0)=$ $S^{\prime}(\bar{u} ; 0)=0$ that

$$
\left\langle-\partial_{u} J(\bar{y}, \bar{u}), h\right\rangle_{U} \leq\left\langle\partial_{y} J(\bar{y}, \bar{u}), G_{\bar{y}}(h)\right\rangle_{V_{\bar{y}}} \leq c\left\|\partial_{y} J(\bar{y}, \bar{u})\right\|_{Y^{*}}\|h\|_{V_{\bar{y}}^{*}}
$$

for all $h \in \operatorname{cone}\left(U_{\text {ad }}-\bar{u}\right)$. Since this set is $V_{\bar{y}}^{*}$-dense in $U$ by Assumption 2.4 and $U \hookrightarrow V_{\bar{y}}^{*}$, this implies the existence of a constant $c>0$ so that

$$
\left|\left\langle-\partial_{u} J(\bar{y}, \bar{u}), h\right\rangle_{U}\right| \leq c\|h\|_{V_{\bar{y}}^{*}} \quad \forall h \in U .
$$

Hence, by the Hahn-Banach theorem, $-\partial_{u} J(\bar{y}, \bar{u})$ can be extended to an element of $V_{\bar{y}}^{* *}$, which we identify with an element $p \in V_{\bar{y}}$ by the reflexivity of $V_{\bar{y}}$. Since $p$ is the extension of $-\partial_{u} J(\bar{y}, \bar{u})$, we immediately deduce (2.4). Inserting this in (2.6) and using that cone $\left(U_{\text {ad }}-\bar{u}\right)$ is $V_{\bar{y}}^{*}$-dense in $U$ and $U$ is dense in $V_{\bar{y}}^{*}$ by Lemma A.1 then gives the second claim.

Based on the previous lemma, we are now in the position to state our main result.

Theorem 2.7 (Strong Stationarity). Let $\bar{u} \in U$ be locally optimal for (P) with associated optimal state $\bar{y}=S(\bar{u})$. Suppose that the Assumptions 2.3 and 2.4 are satisfied at $\bar{u}$. Then there exist $p \in V_{\bar{y}}$ and $\mu \in V_{\bar{y}}^{*}$ so that the following optimality condition is fulfilled:

$$
\begin{gathered}
p+\partial_{u} J(\bar{y}, \bar{u})=0 \quad \text { in } U^{*} \\
A^{*}(\bar{y}) p+\mu=\partial_{y} J(\bar{y}, \bar{u}) \quad \text { in } V_{\bar{y}}^{*}, \\
p \in \mathcal{K}(\bar{y}), \quad\langle\mu, v\rangle_{V_{\bar{y}}} \geq 0 \quad \forall v \in \mathcal{K}(\bar{y}) .
\end{gathered}
$$


Proof. From Lemma 2.6, we already know that there exists a $p \in V_{\bar{y}}$ such that (2.7a) holds. Let us now show that $p \in \mathcal{K}(\bar{y})$. For this purpose, define $\eta:=$ $G_{\bar{y}}(A(\bar{y}) p)$ and $\zeta:=G_{\bar{y}}(A(\bar{y})(p-\eta))$, i.e., $\eta$ and $\zeta$ solve

$$
\begin{aligned}
& \eta \in \mathcal{K}(\bar{y}), \quad\langle A(\bar{y}) \eta, v-\eta\rangle_{V_{\bar{y}}} \geq\langle A(\bar{y}) p, v-\eta\rangle_{V_{\bar{y}}} \quad \forall v \in \mathcal{K}(\bar{y}), \\
& \zeta \in \mathcal{K}(\bar{y}), \quad\langle A(\bar{y}) \zeta, v-\zeta\rangle_{V_{\bar{y}}} \geq\langle A(\bar{y})(p-\eta), v-\zeta\rangle_{V_{\bar{y}}} \quad \forall v \in \mathcal{K}(\bar{y}) .
\end{aligned}
$$

Since $\mathcal{K}(\bar{y})$ is a closed cone by assumption, we can insert $0 \in \mathcal{K}(\bar{y})$ and $2 \eta \in \mathcal{K}(\bar{y})$ as test functions in (2.8), which results in

$$
\langle A(\bar{y})(p-\eta), \eta\rangle_{V_{\bar{y}}}=0 \quad \text { and } \quad\langle A(\bar{y})(p-\eta), v\rangle_{V_{\bar{y}}} \leq 0 \quad \forall v \in \mathcal{K}(\bar{y}) .
$$

The latter inequality implies for (2.9) tested with $v=0$ that $\langle A(\bar{y}) \zeta, \zeta\rangle_{V_{\bar{y}}} \leq 0$, which, thanks to the strong monotonicity of $A(\bar{y})$, in turn gives $\zeta=0$. Next, we insert $h=A(\bar{y})(p-\eta) \in V_{\bar{y}}^{*}$ as a test function in $(2.5)$, which, due to $G_{\bar{y}}(A(\bar{y})(p-$ $\eta))=\zeta=0$, results in

$$
\langle A(\bar{y})(p-\eta), p\rangle_{V_{\bar{y}}} \leq 0 .
$$

Together with the first equation in (2.10), this yields $\langle A(\bar{y})(p-\eta), p-\eta\rangle_{V_{\bar{y}}} \leq 0$ so that the strong monotonicity of $A(\bar{y})$ gives $p=\eta \in \mathcal{K}(\bar{y})$ as claimed.

Next, we simply define $\mu \in V_{\bar{y}}^{*}$ by setting $\mu:=\partial_{y} J(\bar{y}, \bar{u})-A(\bar{y})^{*} p \in V_{\bar{y}}^{*}$ so that $(2.7 \mathrm{~b})$ is fulfilled, too. It remains to verify the last condition in $(2.7 \mathrm{c})$. To this end, let $v \in \mathcal{K}(\bar{y})$ be arbitrary. Then, by construction of $G_{\bar{y}}$, the feasibility of $v$ yields $v=G_{\bar{y}}(A(\bar{y}) v)$. Therefore, if we insert $h=A(\bar{y}) v$ in (2.5), then

$$
\langle\mu, v\rangle_{V_{\bar{y}}}=\left\langle\partial_{y} J(\bar{y}, \bar{u}), v\right\rangle_{V_{\bar{y}}}-\langle A(\bar{y}) v, p\rangle_{V_{\bar{y}}} \geq 0
$$

follows. Since $v \in \mathcal{K}(\bar{y})$ was arbitrary, this completes the proof.

Proposition 2.8. Let Assumption 2.3 hold at a (not necessarily locally optimal) point $\bar{u} \in U_{\mathrm{ad}}$ and assume that $p \in V_{\bar{y}}$ and $\mu \in V_{\bar{y}}^{*}$ exist such that (2.7) is fulfilled. Then $\bar{u}$ satisfies the B-stationarity condition in (2.1).

Proof. Let $h \in \operatorname{cone}\left(U_{\text {ad }}-\bar{u}\right)$ be arbitrary and write again $\delta=S^{\prime}(\bar{u} ; h)$. Similarly to the beginning of the proof of Theorem 2.7, we test the VI in (2.2) with $v=0$ and $v=2 \delta$ to obtain

$$
\langle A(\bar{y}) \delta, v\rangle_{V_{\bar{y}}} \geq(h, v)_{U} \quad \forall v \in \mathcal{K}(\bar{y}) .
$$

Due to $p \in \mathcal{K}(\bar{y})$, this inequality also holds for $v=p$, which, in combination with the adjoint equation in $(2.7 \mathrm{~b})$, gives

$$
\begin{aligned}
\left\langle\partial_{y} J(\bar{y}, \bar{u}), S^{\prime}(\bar{u} ; h)\right\rangle_{Y} & =\left\langle\partial_{y} J(\bar{y}, \bar{u}), \delta\right\rangle_{V_{\bar{y}}} \\
& =\left\langle A(\bar{y})^{*} p+\mu, \delta\right\rangle_{V_{\bar{y}}} \\
& =\langle A(\bar{y}) \delta, p\rangle_{V_{\bar{y}}}+\langle\mu, \delta\rangle_{V_{\bar{y}}} \geq(h, p)_{U} .
\end{aligned}
$$

In view of $(2.7 \mathrm{a})$, this gives the assertion.

Remark 2.9. Theorem 2.7 and Proposition 2.8 demonstrate that, under Assumption 2.3 and the constraint qualification in Assumption 2.4, the optimality system in (2.7) and the B-stationarity condition in (2.1) are equivalent. We have thus 
found an optimality system involving dual variables, which is equivalent to the purely primal optimality condition. This motivates the notion strong stationarity for the optimality condition in (2.7).

Remark 2.10. The result of Theorem 2.7 can be substantially generalized by allowing for a more general structure of the directional derivative of $S$, see [3, Section 6$]$.

\section{Application to Concrete Settings}

\subsection{The Obstacle Problem}

To keep the discussion concise, we restrict ourselves to the classical obstacle problem governed by the Laplacian, i.e.,

$$
y \in K, \quad \int_{\Omega} \nabla y \cdot \nabla(v-y) \mathrm{d} x \geq\langle u, v-y\rangle \quad \forall v \in K .
$$

Herein, $\Omega \subset \mathbb{R}^{d}, d \geq 1$, is a bounded domain and

$$
K:=\left\{v \in H_{0}^{1}(\Omega): \psi_{1} \leq v \leq \psi_{2} \text { a.e. in } \Omega\right\},
$$

with two given functions $\psi_{1}, \psi_{2} \in H^{1}(\Omega)$ such that $K \neq \emptyset$. Clearly, for every $u \in H^{-1}(\Omega),(3.1)$ admits a unique solution $y \in H_{0}^{1}(\Omega)$ and the associated solution operator $S: H^{-1}(\Omega) \rightarrow H_{0}^{1}(\Omega)$ is globally Lipschitz continuous. Moreover, there holds:

Proposition 3.1 ([19, Théorème 3.3]). The solution operator $S$ of (3.1) is directionally differentiable from $H^{-1}(\Omega)$ to $H_{0}^{1}(\Omega)$. Its directional derivative at $u \in H^{-1}(\Omega)$ in direction $h \in H^{-1}(\Omega)$ is given by the unique solution of the following VI of the first kind:

$$
\delta \in \mathcal{K}(y), \quad \int_{\Omega} \nabla \delta \cdot \nabla(v-\delta) \mathrm{d} x \geq\langle h, v-\delta\rangle \quad \forall v \in \mathcal{K}(y),
$$

where $y=S(u)$ and $\mathcal{K}(y) \subset H_{0}^{1}(\Omega)$ is the closed and convex cone defined by

$$
\begin{gathered}
\mathcal{K}(y):=\left\{v \in H_{0}^{1}(\Omega): v \leq 0 \text { q.e., where } y=\psi_{2}, v \geq 0 \text { q.e., where } y=\psi_{1},\right. \\
\langle\Delta y+u, v\rangle=0\} .
\end{gathered}
$$

Note that the pointwise properties in the definition of $\mathcal{K}(y)$ are required quasi-everywhere, i.e., the quasi-continuous representative satisfies the respective property up to sets of zero $H^{1}\left(\mathbb{R}^{d}\right)$-capacity. The proof of the above proposition is based on the polyhedricity of the set $K$, which means that, for all $v \in H_{0}^{1}(\Omega)$ and all $g \in H^{-1}(\Omega)$, there holds

$$
\overline{\operatorname{cone}(K-v)}^{H_{0}^{1}} \cap \operatorname{ker}(g)=\overline{\operatorname{cone}(K-v) \cap \operatorname{ker}(g)} H_{0}^{1},
$$

see $[19,28]$ and $[3$, Section 3.3]. 

follows:

Our optimal control problem governed by the obstacle problem now reads as

$$
\left.\begin{array}{ll}
\min & J(y, u) \\
\text { s.t. } & (y, u) \in H_{0}^{1}(\Omega) \times L^{2}(\Omega), \\
& (y, u) \text { satisfy }(3.1), \quad u \in U_{\mathrm{ad}},
\end{array}\right\}
$$

where $J: H_{0}^{1}(\Omega) \times L^{2}(\Omega) \rightarrow \mathbb{R}$ is a given Fréchet-differentiable objective and $U_{\text {ad }} \subset L^{2}(\Omega)$ is a non-empty, closed, and convex set. Again, we consider a fixed, but arbitrary local minimizer of $\left(\mathrm{P}_{\text {obst }}\right)$, denoted by $(\bar{y}, \bar{u}) \in H_{0}^{1}(\Omega) \times L^{2}(\Omega)$. Then, by setting

$$
U:=L^{2}(\Omega), \quad Y=V_{\bar{y}}:=H_{0}^{1}(\Omega), \quad A(\bar{y}):=-\Delta,
$$

and $\mathcal{K}(\bar{y})$ as defined in $(3.3)$ (with $y=\bar{y})$, problem $\left(\mathrm{P}_{\text {obst }}\right)$ fits into our general setting. Hence, the general theory from Section 2 can be applied to this example:

Theorem 3.2. $\quad$ 1. Suppose that $\bar{u} \in L^{2}(\Omega)$ is locally optimal for $\left(\mathrm{P}_{\mathrm{obst}}\right)$ with associated state $\bar{y}=S(\bar{u})$. Moreover, let the critical constraint qualification in Assumption 2.4 be fulfilled, i.e.,

$$
\overline{\operatorname{cone}\left(U_{\mathrm{ad}}-\bar{u}\right)} L^{2}=L^{2}(\Omega) \text {. }
$$

Then there exist $p \in H_{0}^{1}(\Omega)$ and $\mu \in H^{-1}(\Omega)$ such that

$$
\begin{gathered}
p+\partial_{u} J(\bar{y}, \bar{u})=0 \quad \text { a.e. in } \Omega, \\
-\Delta p+\mu=\partial_{y} J(\bar{y}, \bar{u}) \quad \text { in } H^{-1}(\Omega), \\
p \in \mathcal{K}(\bar{y}), \quad\langle\mu, v\rangle \geq 0 \quad \forall v \in \mathcal{K}(\bar{y}) .
\end{gathered}
$$

2. Assume that $\bar{u} \in U_{\text {ad }}$ with associated state $\bar{y}=S(\bar{u})$ is such that $p \in H_{0}^{1}(\Omega)$ and $\mu \in H^{-1}(\Omega)$ exist so that the system in (3.5) is fulfilled. Then $\bar{u}$ is $B$-stationary for $\left(\mathrm{P}_{\text {obst }}\right)$.

Remark 3.3. The first assertion of Theorem 3.2 concerning the necessary optimality condition was already proven in [20]. There, the critical constraint qualification (3.4) is ensured by simply setting $U_{\text {ad }}=U=L^{2}(\Omega)$.

Another rather implicitly given condition is the assumption that $V_{\bar{y}}=H_{0}^{1}(\Omega)$ must embed into $U=L^{2}(\Omega)$. The injectivity of the embedding operator thus prevents the derivation of strong stationarity conditions in case of boundary controls, where $U=L^{2}(\partial \Omega)$, as the counterexample in [18] demonstrates. Similarly, controls that only act on parts of the domain $\Omega$ can also not be treated by our analysis. This shows that the assumptions concerning the set of admissible controls are indeed rather restrictive.

\subsection{Static Elastoplasticity}

Now we turn to a VI of the second kind and consider an optimal control problem governed by the system of static elastoplasticity with linear kinematic hardening. Strictly speaking, the problem of static elastoplasticity is physically not meaningful, but it may be regarded as the stationary problem that has to be solved 
in one time step of an implicit time discretization of the quasi-static elastoplastic evolution. The model under consideration is the primal formulation of static elastoplasticity with linear kinematic hardening and the von Mises yield condition and reads as follows:

$$
\left.\begin{array}{rl}
(u, p) \in \mathscr{V} \times \mathscr{P} \\
\int_{\Omega}(\varepsilon(u)-p): & \mathbb{C}(\varepsilon(v-u)-(q-p))+p: \mathbb{H}(q-p) \mathrm{d} x \\
+\sigma_{0} & \int_{\Omega}|q|_{F} \mathrm{~d} x-\sigma_{0} \int_{\Omega}|p|_{F} \mathrm{~d} x \\
& \geq\langle\ell, v-u\rangle+\int_{\Omega} \mathscr{L}:(q-p) \mathrm{d} x \quad \forall(v, q) \in \mathscr{V} \times \mathscr{P} .
\end{array}\right\}
$$

Herein, $\Omega \subset \mathbb{R}^{d}, d=2,3$, is a given bounded Lipschitz domain, whose boundary is split into two disjoint parts $\partial \Omega=\Gamma_{D} \cup \Gamma_{N}$. The part $\Gamma_{D}$ is assumed to have positive measure. Moreover, $u: \Omega \rightarrow \mathbb{R}^{d}$ denotes the displacement field, while $p: \Omega \rightarrow \mathbb{R}_{\mathrm{dev}}^{d \times d}$ is the plastic strain tensor. Herein, $\mathbb{R}_{\mathrm{dev}}^{d \times d}$ is the space of symmetric matrices with zero trace, equipped with the Frobenius norm, which is denoted by $|\cdot|_{F}$. The associated scalar product is denoted by $\mathbb{R}^{d \times d} \times \mathbb{R}^{d \times d} \ni(a, b) \mapsto a: b \in \mathbb{R}$. The spaces in (3.6) are defined as follows:

$$
\mathscr{P}:=L^{2}\left(\Omega ; \mathbb{R}_{\mathrm{dev}}^{d \times d}\right), \quad \mathscr{V}:=\left\{v \in W^{1,2}\left(\Omega ; \mathbb{R}^{d}\right): v=0 \text { a.e. on } \Gamma_{D}\right\} .
$$

Moreover, $\mathbb{C}, \mathbb{H} \in L^{\infty}\left(\Omega ; \mathcal{L}\left(\mathbb{R}^{d \times d}, \mathbb{R}^{d \times d}\right)\right)$ are two given symmetric and uniformly coercive mappings (the elasticity and hardening tensor). In addition $\varepsilon=\frac{1}{2}\left(\nabla+\nabla^{\top}\right)$ denotes the linearized strain. Furthermore, $\sigma_{0}>0$ is the uni-axial yield stress, a constant material parameter. There are other equivalent formulations of the system in (3.6), for instance in terms of a VI of the first kind via convex duality, which leads to the so-called dual formulation. A more detailed description of the model can be found in [11].

The variables $\ell \in L^{2}\left(\Omega ; \mathbb{R}^{d}\right)$ and $\mathscr{L} \in \mathscr{P}$ serve as controls in our setting. While $\ell$ has a well defined physical meaning as the loads applied to the body occupying $\Omega$, the physical interpretation of $\mathscr{L}$ is rather critical. It may be seen as a pre-strain, but it is mainly motivated by the mathematical analysis, since it allows us to fulfill Assumption 2.3 in this case, as we will see below.

The optimal control problem considered in this subsection reads as follows:

$$
\begin{array}{cl}
\min & J(u, p, \ell, \mathscr{L}) \\
\text { s.t. } & (u, p, \ell, \mathscr{L}) \in \mathscr{V} \times \mathscr{P} \times L^{2}\left(\Omega ; \mathbb{R}^{d}\right) \times \mathscr{P} \\
& (u, p, \ell, \mathscr{L}) \text { satisfy }(3.6)
\end{array}
$$

with a Fréchet-differentiable objective $J: \mathscr{V} \times \mathscr{P} \times L^{2}\left(\Omega ; \mathbb{R}^{d}\right) \times \mathscr{P} \rightarrow \mathbb{R}$. We could also consider additional control constraints (and would then again need an additional assumption of the form in Assumption 2.4), but, in order to keep the discussion concise, we restrict to the case without control constraints. 
Thanks to Korn's inequality and the coercivity of $\mathbb{C}$ and $\mathbb{H}$, the VI in (3.6) admits a unique solution in $\mathscr{V} \times \mathscr{P}$ for every right-hand side in $\mathscr{V}^{*} \times \mathscr{P}$, see, e.g., [11]. The next result shows that the associated solution mapping is directionally differentiable and was established in [3, Section 4.3]:

Proposition 3.4 ([3, Corollary 4.3.5]). The solution operator of (3.6), denoted by $S:(\ell, \mathscr{L}) \mapsto(u, p)$, is directionally differentiable from $\mathscr{V}^{*} \times \mathscr{P}$ to $\mathscr{V} \times \mathscr{P}$. Its directional derivative at $(\ell, \mathscr{L}) \in \mathscr{V}^{*} \times \mathscr{P}$ in direction $(h, G) \in \mathscr{V}^{*} \times \mathscr{P}$ is given by the unique solution $\left(u^{\prime}, p^{\prime}\right)$ of

$$
\begin{aligned}
& \left(u^{\prime}, p^{\prime}\right) \in \mathscr{V} \times K(u, p), \\
& \left\langle\mathcal{A}(p)\left(u^{\prime}, p^{\prime}\right),(v, q)-\left(u^{\prime}, p^{\prime}\right)\right\rangle \\
& \quad \geq\left\langle h, v-u^{\prime}\right\rangle+\int_{\Omega} G:\left(q-p^{\prime}\right) \mathrm{d} x \quad \forall(v, q) \in \mathscr{V} \times K(u, p),
\end{aligned}
$$

where $(u, p)=S(\ell, \mathscr{L})$,

$$
\begin{aligned}
\mathscr{P}_{p} & :=\left\{q \in \mathscr{P}: \int_{\{p \neq 0\}}|p|_{F}^{-3}\left(|p|_{F}^{2}|q|_{F}^{2}-(p: q)^{2}\right) \mathrm{d} x<\infty\right\}, \\
K(u, p) & :=\left\{q \in \mathscr{P}_{p}:(\mathscr{L}+\mathbb{C} \varepsilon(u)): q=\sigma_{0}|q|_{F} \text { a.e., where } p=0\right\},
\end{aligned}
$$

and

$$
\begin{aligned}
& \mathcal{A}(p): \mathscr{V} \times \mathscr{P}_{p} \rightarrow \mathscr{V}^{*} \times \mathscr{P}_{p}^{*} \\
& \begin{aligned}
\langle\mathcal{A}(p)(w, r),(v, q)\rangle:= & \int_{\Omega}(\varepsilon(w)-r): \mathbb{C}(\varepsilon(v)-q)+r: \mathbb{H} q \mathrm{~d} x \\
& \quad+\sigma_{0} \int_{\{p \neq 0\}}|p|_{F}^{-3}\left(|p|_{F}^{2} r: q-(p: r)(p: q)\right) \mathrm{d} x .
\end{aligned}
\end{aligned}
$$

The directional differentiability of the solution operator associated with the (equivalent) dual formulation of (3.6) was investigated in [1, 14]. Via convex duality, one shows that these results are in accordance with the above proposition.

Let us now again consider a fixed, but arbitrary local minimizer of $\left(\mathrm{P}_{\text {plast }}\right)$, denoted by $(\bar{\ell}, \overline{\mathscr{L}}) \in L^{2}\left(\Omega ; \mathbb{R}^{d}\right) \times \mathscr{P}$ with associated state $\bar{y}:=(\bar{u}, \bar{p})=S(\bar{\ell}, \overline{\mathscr{L}})$. Then with the setting

$$
\begin{gathered}
U=L^{2}\left(\Omega ; \mathbb{R}^{d}\right) \times \mathscr{P}, \quad Y=\mathscr{V} \times \mathscr{P}, \quad V_{\bar{y}}:=\mathscr{V} \times \mathscr{P}_{\bar{p}}, \\
\mathcal{K}(\bar{y}):=\mathscr{V} \times K(\bar{u}, \bar{p}), \quad A(\bar{y}):=\mathcal{A}(\bar{p}),
\end{gathered}
$$

the problem of static elastoplasticity fits into our general framework, as we will see in the following. Equipped with the scalar product

$$
((w, r),(v, q))_{V_{\bar{y}}}:=(w, v)_{\mathscr{V}}+(r, q)_{\mathscr{P}}+\int_{\{\bar{p} \neq 0\}}|\bar{p}|_{F}^{-3}\left(|\bar{p}|_{F}^{2} r: q-(\bar{p}: r)(\bar{p}: q)\right) \mathrm{d} x,
$$

the space $V_{\bar{y}}$ becomes a Hilbert space as required. Since $\mathscr{P}_{\bar{p}}$ is a dense subset of $\mathscr{P}, V_{\bar{y}}$ is dense in $U$. We point out that the presence of the additional (and rather artificial) control variable $\mathscr{L}$ is crucial for the embedding of $V_{\bar{y}}$ in $U$ (with the injective pointwise identity as embedding operator). Furthermore, by using Korn's 
inequality, one shows that $A(\bar{y})=\mathcal{A}(\bar{p})$ as defined in (3.7) is strongly monotone. Therefore, all conditions in Assumption 2.3 are fulfilled. Since, in addition, Assumption 2.4 is trivially satisfied as $U_{\text {ad }}=U$, one deduces the following

Theorem 3.5 ([3, Corollary 6.1.13]). $\quad$ 1. Let $(\bar{\ell}, \overline{\mathscr{L}}) \in L^{2}\left(\Omega ; \mathbb{R}^{d}\right) \times \mathscr{P}$ with associated state $\bar{y}=(\bar{u}, \bar{p}) \in \mathscr{V} \times \mathscr{P}$ be locally optimal for $\left(\mathrm{P}_{\text {plast }}\right)$. Then there exist an adjoint state $(w, r) \in \mathscr{V} \times \mathscr{P}_{\bar{p}}$ and a multiplier $\mu \in \mathscr{P}$ such that

$$
\begin{aligned}
w+\partial_{\ell} J(\bar{u}, \bar{p}, \bar{\ell}, \overline{\mathscr{L}})=0 & \text { a.e. in } \Omega, \\
r+\partial_{\mathscr{L}} J(\bar{u}, \bar{p}, \bar{\ell}, \overline{\mathscr{L}})=0 & \text { a.e. in } \Omega,
\end{aligned}
$$

$$
\left.\begin{array}{r}
\langle\mathcal{A}(\bar{p})(w, r),(v, q)\rangle \\
=\left\langle\partial_{u} J(\bar{u}, \bar{p}, \bar{\ell}, \overline{\mathscr{L}}), v\right\rangle_{\mathscr{V}}+\int_{\Omega} \partial_{p} J(\bar{u}, \bar{p}, \bar{\ell}, \overline{\mathscr{L}}): q \mathrm{~d} x \\
-\int_{\Omega} \mu: q \mathrm{~d} x \quad \forall(v, q) \in \mathscr{V} \times \mathscr{P}_{\bar{p}}
\end{array}\right\}
$$

2. If a couple $(\bar{\ell}, \overline{\mathscr{L}}) \in L^{2}\left(\Omega ; \mathbb{R}^{d}\right) \times \mathscr{P}$ together with its associated state $(\bar{u}, \bar{p})=$ $S(\bar{\ell}, \overline{\mathscr{L}})$, an adjoint state $(w, r) \in \mathscr{V} \times \mathscr{P}_{\bar{p}}$ and a multiplier $\mu \in \mathscr{P}$ satisfies the system (3.8), then it is B-stationary for ( $\left.\mathrm{P}_{\text {plast }}\right)$.

Remark 3.6. It is noteworthy that, in this example in contrast to the previous one in Section 3.1, the space $V_{\bar{y}}$ as well as the operator $A(\bar{y})$ differ from the original state space $Y$ and the "smooth part" in the VI in (3.6) associated with the controlto-state map. This effect will also appear in the following two examples in the next sections.

Let us finally remark that a strong stationarity system for optimal control of static plasticity in dual, i.e., stress-based formulation is derived in [14] under slightly more restrictive assumptions.

\subsection{The Lasso Problem in Sobolev Spaces}

This subsection is devoted to an optimal control problem governed by the following VI of the second kind:

$$
\begin{aligned}
& y \in H_{0}^{1}(\Omega), \\
& \int_{\Omega} \nabla y \cdot \nabla(v-y) \mathrm{d} x+\|v\|_{L^{1}(\Omega)}-\|y\|_{L^{1}(\Omega)} \geq\langle u, v-y\rangle \quad \forall v \in H_{0}^{1}(\Omega),
\end{aligned}
$$

where $\Omega \subset \mathbb{R}^{d}, d \geq 2$, is a bounded Lipschitz domain in the sense of $[10$, Definition 4.4]. In finite dimensions, VIs of this type arise in the context of sparse linear regression and are occasionally called lasso problem, see, e.g., [24]. By the 
direct method of the calculus of variations, one shows that, for every right-hand side $u \in H^{-1}(\Omega)$, there exists a unique solution $y \in H_{0}^{1}(\Omega)$ of $(3.9)$ and the associated solution operator, denoted by $S: H^{-1}(\Omega) \rightarrow H_{0}^{1}(\Omega)$, is globally Lipschitz. Its differentiability properties however constitute a delicate issue. Via convex duality, one can transform the VI in (3.9) into an equivalent obstacle problem in $H^{-1}(\Omega)$. However, its feasible set given by

$$
\begin{array}{r}
\Lambda:=\left\{\lambda \in H^{-1}(\Omega): \exists q \in L^{\infty}(\Omega) \text { such that }|q| \leq 1 \text { a.e. in } \Omega\right. \\
\text { and } \left.\langle\lambda, v\rangle=\int_{\Omega} q v \mathrm{~d} x\right\}
\end{array}
$$

is in general not polyhedric, as the counterexamples in $[5,6,28]$ demonstrate. If one assumes that this set is polyhedric, then the analysis of $[12,19]$ can be adapted to prove the directional differentiability of $S$, see $[17,23]$. In [9] comparatively restrictive conditions are established which guarantee that this set behaves like a polyhedric set. Another approach that goes without polyhedricity is pursued in [5] and yields the following result:

Proposition 3.7 ([5], [3, Theorem 5.2.15]). Let $u \in H^{-1}(\Omega)$ be given with associated state $y=H_{0}^{1}(\Omega)$ and suppose that the following assumptions are fulfilled:

- (Regularity) It holds $y \in C^{1}(\Omega) \cap H_{0}^{1}(\Omega)$.

- (Structure of the Active Set) There exists a set $\mathcal{C} \subseteq \partial\{y \neq 0\} \cup \partial \Omega$ such that

1. $\mathcal{C}$ is closed and has $H^{1}\left(\mathbb{R}^{d}\right)$-capacity zero,

2. $(\partial\{y \neq 0\} \cup \partial \Omega) \backslash \mathcal{C}$ is a (strong) $(d-1)$-dimensional Lipschitz submanifold of $\mathbb{R}^{d}$,

3. the sets

$$
\begin{aligned}
& \mathcal{N}_{+}:=\{\nabla y=0\} \cap \partial\{y>0\} \backslash \mathcal{C}, \quad \mathcal{N}_{-}:=\{\nabla y=0\} \cap \partial\{y<0\} \backslash \mathcal{C} \\
& \text { are relatively open in }(\partial\{y \neq 0\} \cup \partial \Omega) \backslash \mathcal{C} .
\end{aligned}
$$

Then $S$ is directionally differentiable at $u$ in every direction $h \in H^{-1}(\Omega)$ and the directional derivative $\delta=S^{\prime}(u ; h)$ is given by the unique solution of the following VI of the first kind:

$$
\begin{aligned}
& \delta \in \mathcal{K}(y), \\
& \int_{\Omega} \nabla \delta \cdot \nabla(v-\delta) \mathrm{d} x+2 \int_{\mathcal{M}} \frac{\tau(\delta) \tau(v-\delta)}{\|\nabla y\|_{2}} \mathrm{~d} \mathcal{H}^{d-1} \geq\langle h, v-\delta\rangle \quad \forall v \in \mathcal{K}(y),
\end{aligned}
$$

where $\mathcal{M}:=\{y=0\} \cap\{\nabla y \neq 0\}$ and $\tau$ is the associated trace operator. Furthermore, the convex cone $\mathcal{K}(y)$ is given by

$$
\begin{array}{r}
\mathcal{K}(y):=\left\{v \in H_{0}^{1}(\Omega): \tau(v)^{-}=0 \text { a.e. on } \mathcal{N}_{+}, \tau(v)^{+}=0 \text { a.e. on } \mathcal{N}_{-},\right. \\
\left.|v|=\lambda v \text { a.e. in }\{y=0\}, \int_{\mathcal{M}} \frac{\tau(v)^{2}}{\|\nabla y\|_{2}} \mathrm{~d} \mathcal{H}^{d-1}<\infty\right\},
\end{array}
$$

where $\lambda \in L^{\infty}(\Omega)$ is the unique element of $\partial\|\cdot\|_{L^{1}(\Omega)}(\bar{y})$ that satisfies $\lambda=u+\Delta y$. 
Note that the sets $\mathcal{C}, \mathcal{N}_{ \pm}$, and $\mathcal{M}$ depend on the solution $y=S(u)$, but we suppress this dependency in order to simplify the notation.

Remark 3.8. It is to be noted that, in contrast to the previous examples in Sections 3.1 and 3.2, there is - to the best of our knowledge - no result available in the literature that guarantees the directional differentiability of the solution operator to (3.9) without further assumptions. The assumptions in Proposition 3.7 are on the one hand easily verifiable, once the solution $y$ is known (in contrast to the polyhedricity of $\Lambda$ ), and on the other hand substantially less restrictive compared to the assumptions in [9]. Proposition 3.7 therefore can be seen as the most rigorous differentiability result for the solution operator of (3.9).

Remark 3.9. The lack of polyhedricity of $\Lambda$ is also illustrated by the integral over the set $\mathcal{M}$ in (3.10), which does not appear, if the set $\Lambda$ is polyhedric, see $[9,17]$. This integral is closely related to the pull-back of the second distributional derivative of the absolute value function, see [3, Section 5.2.2] for details.

Similarly to the previous examples, we consider an optimal control problem of the form

$$
\left.\begin{array}{ll}
\min & J(y, u) \\
\text { s.t. } & (y, u) \in H_{0}^{1}(\Omega) \times L^{2}(\Omega), \\
& (y, u) \text { satisfy }(3.9),
\end{array}\right\} \quad\left(\mathrm{P}_{\text {lasso }}\right)
$$

where $J: H_{0}^{1}(\Omega) \times L^{2}(\Omega) \rightarrow \mathbb{R}$ is a given Fréchet-differentiable objective. To keep the discussion concise, we again set $U_{\text {ad }}=L^{2}(\Omega)$ in order to fulfill the constraint qualification in Assumption 2.4. As before, we consider a fixed, but arbitrary local minimizer of $\left(\mathrm{P}_{\text {lasso }}\right)$, denoted by $(\bar{y}, \bar{u}) \in H_{0}^{1}(\Omega) \times L^{2}(\Omega)$. If we set

$$
\begin{gathered}
U:=L^{2}(\Omega), \quad Y:=H_{0}^{1}(\Omega), \quad V_{\bar{y}}:=\left\{v \in H_{0}^{1}(\Omega): \int_{\mathcal{M}} \frac{\tau(v)^{2}}{\|\nabla \bar{y}\|_{2}} \mathrm{~d} \mathcal{H}^{d-1}<\infty\right\}, \\
\langle A(\bar{y}) w, v\rangle:=\int_{\Omega} \nabla w \cdot \nabla v \mathrm{~d} x+2 \int_{\mathcal{M}} \frac{\tau(w) \tau(v)}{\|\nabla \bar{y}\|_{2}} \mathrm{~d} \mathcal{H}^{d-1}, \quad v, w \in V_{\bar{y}},
\end{gathered}
$$

and $\mathcal{K}(\bar{y})$ as defined in $(3.11)$ (with $y=\bar{y}$ ), then ( $\left.\mathrm{P}_{\text {lasso }}\right)$ fits into our general setting. With the obvious scalar product

$$
(w, v)_{V_{\bar{y}^{*}}}:=\langle A(\bar{y}) w, v\rangle,
$$

$V_{\bar{y}}$ becomes a Hilbert space and $A(\bar{y})$ is clearly strongly monotone in this space. Moreover, $\mathcal{K}(\bar{y})$ is closed in this space. Hence, the general theory from Section 2 is applicable and yields:

Theorem 3.10. $\quad$ 1. Suppose that $\bar{u} \in L^{2}(\Omega)$ with associated state $\bar{y}=S(\bar{u})$ is locally optimal for $\left(\mathrm{P}_{\text {lasso }}\right)$ and assume moreover that $\bar{y}$ is such that the assumptions of Proposition 3.7 on the regularity of $\bar{y}$ and the structure of its active set are fulfilled (with $y=\bar{y}$ ). Then there exist an adjoint state $p \in V_{\bar{y}}$ 
and a multiplier $\mu \in V_{\bar{y}}^{*}$ such that

$$
\begin{gathered}
\left.\begin{array}{c}
p+\partial_{u} J(\bar{y}, \bar{u})=0 \quad \text { a.e. in } \Omega, \\
\int_{\Omega} \nabla p \cdot \nabla v \mathrm{~d} x+2 \int_{\mathcal{M}} \frac{\tau(p) \tau(v)}{\|\nabla \bar{y}\|_{2}} \mathrm{~d} \mathcal{H}^{d-1} \\
=\left\langle\partial_{y} J(\bar{y}, \bar{u}), v\right\rangle_{H_{0}^{1}(\Omega)}-\langle\mu, v\rangle_{V_{\bar{y}}}, \quad \forall v \in V_{\bar{y}}, \quad \\
p \in \mathcal{K}(\bar{y}), \quad\langle\mu, v\rangle_{V_{\bar{y}}} \geq 0 \quad \forall v \in \mathcal{K}(\bar{y}) .
\end{array}\right\}
\end{gathered}
$$

2. Let $\bar{u} \in L^{2}(\Omega)$ be given such that its state $\bar{y}=S(\bar{u})$ satisfies the assumptions in Proposition 3.7 (with $y=\bar{y}$ ). If an adjoint state $p \in V_{\bar{y}}$ and a multiplier $\mu \in V_{\bar{y}}^{*}$ exist such that (3.12) holds true, then $\bar{u}$ is B-stationary for ( $\left.\mathrm{P}_{\text {lasso }}\right)$.

Again we observe that the space $V_{\bar{y}}$ differs from the original state space $Y$ and the bilinear form of the adjoint equation differs from the one in the VI defining the control-to-state map, similarly to the elastoplastic system in the previous section.

\subsection{Non-Newtonian Fluids - the Mosolov Problem}

Our last example arises in the modeling of non-Newtonian fluids. To be more precise, we consider the so-called Mosolov problem, which models the steady-state motion of a viscoplastic fluid in a cylindrical pipe of cross-section $\Omega \subset \mathbb{R}^{2}$ under no-slip boundary conditions, see [21] for details on the physical background. After setting all material parameters to one, the model is similar to the lasso problem and reads

$$
\begin{aligned}
& y \in H_{0}^{1}(\Omega), \\
& \int_{\Omega} \nabla y \cdot \nabla(v-y) \mathrm{d} x+\int_{\Omega}|\nabla v| \mathrm{d} x-\int_{\Omega}|\nabla y| \mathrm{d} x \geq\langle u, v-y\rangle \quad \forall v \in H_{0}^{1}(\Omega),
\end{aligned}
$$

where $\Omega \subset \mathbb{R}^{2}$ is a bounded and simply connected Lipschitz domain and $|\cdot|$ denotes the Euclidean norm of a vector. The state variable $y: \Omega \rightarrow \mathbb{R}$ describes the velocity of the fluid in direction of the pipe (i.e., perpendicular to $\Omega$ ) and $u: \Omega \rightarrow \mathbb{R}$ is a volume force acting in this direction. The restriction to the two-dimensional setting is on the one hand motivated by the application background and on the other hand essential for the mathematical analysis presented in the following.

Again, the existence and uniqueness for (3.13) follow immediately from the direct method of the calculus of variations. The associated solution map $S$ is globally Lipschitz from $H^{-1}(\Omega)$ to $H_{0}^{1}(\Omega)$. However, as in case of the lasso problem, the directional differentiability of $S$ is a challenging issue. As it turns out, we again need additional assumptions on the respective state to obtain the existence of a directional derivative. Unfortunately, these assumptions are not as easily checked as in case of the lasso problem. In order to formulate these assumptions, we need to define the following sets. Given the solution $y \in H_{0}^{1}(\Omega)$ of $(3.13)$ and assuming that this solution admits a continuously differentiable representative, we introduce 
the active, inactive, and biactive sets as follows:

$$
\begin{aligned}
\mathcal{I} & :=\{|\nabla y|>0\}, \quad \mathcal{A}:=\{|\nabla y|=0\}, \quad \mathcal{A}^{\circ}:=\operatorname{int}(\mathcal{A}), \quad \mathcal{B}:=\partial \mathcal{A} \cup \partial \Omega, \\
\mathcal{B}^{\circ}:=\{x \in \partial \mathcal{A}: & \text { there exists an open neighborhood } D \subseteq \Omega \text { of } x \text { such that } \\
& D \cap \partial \mathcal{A} \text { is a one-dimensional } C^{1} \text {-submanifold of } \mathbb{R}^{2} \\
& \text { and such that } D \cap \partial \mathcal{A}=D \cap \partial\{y=c\} \text { for some } c \in \mathbb{R}\} .
\end{aligned}
$$

Of course, these sets depend on the respective solution $y$, but we suppress this dependency for the ease of notation. Moreover, given a set $\mathcal{M} \subset \Omega$, we denote the set of all connected components of $\mathcal{M}$ by $\left\{\mathcal{M}_{i}\right\}$.

Proposition 3.11 ([3, Theorem 5.1.37]). Let $u \in H^{-1}(\Omega)$ be given and suppose that the associated state $y=S(u)$ satisfies the following hypotheses:

- (Regularity) It holds $y \in C^{1,1}(\Omega) \cap H_{0}^{1}(\Omega)$ and $\Delta y+u \in L^{\infty}(\Omega)$.

- (Structure of the Active and the Inactive Set)

1. the collections $\left\{\mathcal{I}_{i}\right\},\left\{\mathcal{A}_{i}\right\},\left\{\mathcal{A}_{i}^{\circ}\right\},\left\{\mathcal{B}_{i}\right\}$ are finite,

2. the components $\mathcal{A}_{i}^{\circ}$ and $\mathcal{I}_{i}$ are Lipschitz domains for all $i$,

3. the components $\mathcal{A}_{i}$ and $\mathcal{B}_{i}$ are Lipschitz connected for all $i$,

4. the set $\overline{\mathcal{B}^{\circ}} \backslash \mathcal{B}^{\circ}$ is finite and $\mathcal{B}=\overline{\mathcal{B}^{\circ}} \cup \partial \Omega$.

- (Well-Behavedness of the Normalized Gradient Field) There exist a function $\omega \in C^{0,1}(\Omega)$, a constant $C>0$, and an open set $D \subseteq \mathbb{R}^{2}$ with $\mathcal{A} \cup \partial \Omega \subseteq D$ and

$$
\begin{gathered}
\omega=0 \text { on } \mathcal{A} \cup \partial \Omega, \quad \operatorname{dist}(\cdot, \mathcal{A} \cup \partial \Omega) \leq C \omega \text { a.e. in } \mathcal{I} \cap D, \\
\left(\frac{\nabla y^{\perp}}{|\nabla y|} \cdot \frac{\nabla \omega}{|\nabla \omega|}\right)^{2} \leq C|\nabla y| \text { a.e. in } \mathcal{I} \cap D,
\end{gathered}
$$

where, here and in all what follows, $(a, b)^{\perp}=(b,-a)$ for $a, b \in \mathbb{R}$.

Under these assumptions, the solution operator $S$ of (3.13) is directionally differentiable at $u$ in every direction $h \in H^{-1}(\Omega)$ and the directional derivative $\delta:=S^{\prime}(u ; h)$ is uniquely characterized by the following VI of the first kind $\delta \in \mathcal{K}(y)$

$\int_{\Omega} \nabla \delta \cdot \nabla(v-\delta) \mathrm{d} x+\int_{\mathcal{I}} \frac{\left(\nabla y^{\perp} \cdot \nabla \delta\right)\left(\nabla y^{\perp} \cdot \nabla(v-\delta)\right)}{|\nabla y|^{3}} \mathrm{~d} x \geq\langle h, v-\delta\rangle \quad \forall v \in \mathcal{K}(y)$, where the convex cone $\mathcal{K}(y)$ is given by

$$
\mathcal{K}(y):=\left\{v \in H_{0}^{1}(\Omega): \int_{\mathcal{I}} \frac{\left(\nabla y^{\perp} \cdot \nabla v\right)^{2}}{|\nabla y|^{3}} \mathrm{~d} x<\infty,|\nabla v|=\lambda \cdot \nabla v \text { a.e. in } \mathcal{A}\right\},
$$

where $\lambda \in L^{\infty}\left(\Omega ; \mathbb{R}^{2}\right)$ is any element of $\partial\|\cdot\|_{L^{1}\left(\Omega ; \mathbb{R}^{2}\right)}(\nabla y)$ that satisfies $\operatorname{div} \lambda=$ $u+\Delta y$ in $H^{-1}(\Omega)$.

Remark 3.12. Some words concerning the above proposition are in order. First of all, the existence of $\lambda$ simply follows from the reformulation of the VI in (3.13) by means of the chain rule for convex subdifferentials, and it is easily shown that, for 
every such $\lambda$, the set $\mathcal{K}(y)$ is the same. Moreover, while the regularity assumptions as well as the structural assumptions on the active and inactive sets can directly be checked, if a solution $y$ is given, the intrinsic third assumption is hard to verify in practice, see [3, Section 5.1.5] for details. Finally, for the notion of Lipschitz connected sets, we refer to [3, Definition 5.1.24].

Similarly to the previous examples, the optimal control problem associated with (3.13) reads

$$
\left.\begin{array}{ll}
\min & J(y, u) \\
\text { s.t. } & (y, u) \in H_{0}^{1}(\Omega) \times L^{2}(\Omega), \\
& (y, u) \text { satisfy }(3.13),
\end{array}\right\} \quad\left(\mathrm{P}_{\text {moso }}\right)
$$

where $J: H_{0}^{1}(\Omega) \times L^{2}(\Omega) \rightarrow \mathbb{R}$ is a given Fréchet-differentiable objective. Again, we set $U_{\mathrm{ad}}=L^{2}(\Omega)$ so that the constraint qualification in Assumption 2.4 is automatically fulfilled. As before, we consider a fixed, but arbitrary local minimizer $\bar{u} \in L^{2}(\Omega)$ with associated state $\bar{y} \in H_{0}^{1}(\Omega)$. This time we set

$$
\begin{gathered}
U:=L^{2}(\Omega), \quad Y:=H_{0}^{1}(\Omega), \quad V_{\bar{y}}:=\left\{v \in H_{0}^{1}(\Omega): \int_{\mathcal{I}} \frac{\left(\nabla \bar{y}^{\perp} \cdot \nabla v\right)^{2}}{|\nabla \bar{y}|^{3}} \mathrm{~d} x<\infty\right\}, \\
\langle A(\bar{y}) w, v\rangle:=\int_{\Omega} \nabla w \cdot \nabla v \mathrm{~d} x+\int_{\mathcal{I}} \frac{\left(\nabla \bar{y}^{\perp} \cdot \nabla w\right)\left(\nabla \bar{y}^{\perp} \cdot \nabla v\right)}{|\nabla \bar{y}|^{3}} \mathrm{~d} x, \quad v, w \in V_{\bar{y}}
\end{gathered}
$$

and $\mathcal{K}(\bar{y})$ as defined in (3.14) (with $y=\bar{y}$ and $\lambda$ associated with $\bar{y}$ ). As in case of the lasso problem, $V_{\bar{y}}$ becomes a Hilbert space if endowed with the scalar product $(v, w)_{V_{\bar{y}}}:=\langle A(\bar{y}) v, w\rangle$ so that $A(\bar{y})$ is automatically strongly monotone and $\mathcal{K}(\bar{y})$ is closed in this space. Hence, we can again apply the general theory, which results in the following

Theorem 3.13. $\quad$ 1. Let $\bar{u} \in L^{2}(\Omega)$ be locally optimal for $\left(\mathrm{P}_{\text {moso }}\right)$ and assume that the associated state $\bar{y}=S(\bar{u})$ satisfies the assumptions in Proposition 3.11 (with $y=\bar{y}$ ). Then there exist an adjoint state $p \in V_{\bar{y}}$ and a multiplier $\mu \in V_{\bar{y}}^{*}$ such that the following optimality conditions are fulfilled:

$$
\begin{aligned}
& p+\partial_{u} J(\bar{y}, \bar{u})=0 \quad \text { a.e. in } \Omega, \\
& \left.\begin{array}{rl}
\int_{\Omega} \nabla p \cdot \nabla v \mathrm{~d} x & +\int_{\mathcal{I}} \frac{\left(\nabla \bar{y}^{\perp} \cdot \nabla p\right)\left(\nabla \bar{y}^{\perp} \cdot \nabla v\right)}{|\nabla \bar{y}|^{3}} \mathrm{~d} x \\
& =\left\langle\partial_{y} J(\bar{y}, \bar{u}), v\right\rangle_{H_{0}^{1}(\Omega)}-\langle\mu, v\rangle_{V_{\bar{y}}}, \quad \forall v \in V_{\bar{y}},
\end{array}\right\} \\
& p \in \mathcal{K}(\bar{y}), \quad\langle\mu, v\rangle_{V_{\bar{y}}} \geq 0 \quad \forall v \in \mathcal{K}(\bar{y}) .
\end{aligned}
$$

2. If $\bar{u} \in L^{2}(\Omega)$ is such that $\bar{y}=S(\bar{u})$ fulfills the assumptions in Proposition 3.11 and there exist $p \in V_{\bar{y}}$ and $\mu \in V_{\bar{y}}^{*}$, then $\bar{u}$ is B-stationary for $\left(\mathrm{P}_{\text {moso }}\right)$.

\section{Conclusion}

Within this article, we constructed a general framework for the derivation of strong stationarity conditions for optimal control problems governed by VIs. Moreover, we 
demonstrated by means of application-driven examples that our general analysis also applies in case of VIs of the second kind. However, as our two last examples show, sometimes additional assumptions, that may even be hard to verify, are necessary to guarantee that the control-to-state map associated with the VI under consideration is directionally differentiable and the directional derivatives possess the desired structure. Under these assumptions, though, our general framework is applicable and yields stationarity conditions, which are the most rigorous possible ones.

It is however an open question how to solve these strong stationarity systems numerically. The reason is that, in neither of our four examples, the adjoint equation together with the (generalized) sign conditions on the adjoint state and the multiplier $\mu$ forms a VI or a complementarity system, which would be amenable for numerical computations. Even worse, as the investigations on optimal control of non-smooth PDEs in [4] show, strong stationarity systems may be potentially overdetermined. The construction of algorithms for the reliable numerical computation of strongly stationary points is therefore an ongoing field of future research.

\section{Appendix A. Auxiliary Results}

Lemma A.1. Under Assumption 2.3, $U$ is dense in $V_{\bar{y}}^{*}$.

Proof. Let us assume that $U$ is not a dense subset of $V_{\bar{y}}^{*}$ so that there exists a $g \in V_{\bar{y}}^{*} \backslash \bar{U}^{V_{\bar{y}}^{*}}$. Then the strict separation theorem in combination with the reflexivity of $V_{\bar{y}}$ implies the existence of a $v \in V_{\bar{y}}, v \neq 0$, such that

$$
(h, v)_{U}=0<\langle g, v\rangle_{V_{\bar{y}}} \quad \forall h \in U .
$$

Since $V_{\bar{y}} \hookrightarrow U$ and the embedding is injective, this yields $v=0$, which is a contradiction.

\section{References}

[1] T. Betz, Optimal control of two variational inequalities arising in solid mechanics. PhD thesis, Technische Universität Dortmund, 2015.

[2] J. F. Bonnans and A. Shapiro, Perturbation Analysis of Optimization Problems. Springer, 2000.

[3] C. Christof, Sensitivity Analysis of Elliptic Variational Inequalities of the First and the Second Kind. PhD thesis, Technische Universität Dortmund, 2018.

[4] C. Christof, C. Clason, C. Meyer, and S. Walther, Optimal control of a non-smooth semilinear elliptic equation. Math. Control and Related Fields 8 (2018), 247-276.

[5] C. Christof and C. Meyer, Sensitivity analysis for a class of $H_{0}^{1}$-elliptic variational inequalities of the second kind. Set-Valued and Variational Analysis 27 (2019), 469502.

[6] C. Christof and G. Wachsmuth, On the Non-Polyhedricity of Sets with Upper and Lower Bounds in Dual Spaces. GAMM Reports 40 (2018), 339-350. 
[7] J. C. de los Reyes, Optimization of mixed variational inequalities arising in flow of viscoplastic materials. COAP 52 (2012), 757-784.

[8] J. C. de los Reyes, On the optimal control of some nonsmooth distributed parameter systems arising in mechanics. GAMM Reports 40 (2017), 268-286.

[9] J. C. de los Reyes and C. Meyer, Strong stationarity conditions for a class of optimization problems governed by variational inequalities of the second kind. J. Optim. Theory Appl. 168 (2016), 375-409.

[10] L. C. Evans and R. F. Gariepy, Measure Theory and Fine Properties of Functions. CRC Press, 2015.

[11] W. Han and B. D. Reddy, Plasticity: Mathematical Theory and Numerical Analysis. Springer, 1999.

[12] A. Haraux, How to differentiate the projection on a convex set in Hilbert space. Some applications to variational inequalities. J. Math. Soc. Japan 29 (1977), 615-631.

[13] R. Herzog and C. Meyer, Optimal control of static plasticity with linear kinematic hardening. ZAMM 91 (2011), 777-794.

[14] R. Herzog, C. Meyer and G. Wachsmuth, B- and strong stationarity for optimal control of static plasticity with hardening. SIAM J. Optim. 23 (2013), 321-352.

[15] M. Hintermüller and I. Kopacka, Mathematical programs with complementarity constraints in function space: $C$ - and strong stationarity and a path-following algorithm. SIAM Journal on Optimization 20 (2009), 86-902.

[16] M. Hintermüller and T. Surowiec, First-Order Optimality Conditions for Elliptic Mathematical Programs with Equilibrium Constraints via Variational Analysis. SIAM Journal on Optimization 21 (2011), 1561-1593.

[17] M. Hintermüller and T. Surowiec, On the Directional Differentiability of the Solution Mapping for a Class of Variational Inequalities of the Second Kind. Set-Valued and Variational Analysis 26 (2018), 631-642.

[18] C. Meyer and D. Wachsmuth, Strong stationarity is not a necessary optimality condition for boundary control of the obstacle problem. Preprint Nr. 327, Ergebnisberichte des Instituts für Angewandte Mathematik, Universität Würzburg, 2014.

[19] F. Mignot, Contrôle dans les inéquations variationelles elliptiques. J. Funct. Anal. 22 (1976), 130-185.

[20] F. Mignot and J. P. Puel, Optimal control in some variational inequalities. SIAM J. Control Optim. 22 (1984), 466-476.

[21] P. P. Mosolov and V. P. Miasnikov, Variational methods in the theory of the fluidity of a viscousplastic medium. J. Appl. Math. Mech. 29 (1965), 545-577.

[22] B. Schweizer, Partielle Differentialgleichungen. Springer, 2013.

[23] J. Sokołowski, Sensitivity analysis of contact problems with prescribed friction. Appl. Math. Optim. 18 (1988), 99-117.

[24] R. Tibshirani, Regression Analysis and Selection via the Lasso. Royal Statistical Society Series 58 (1996) 267-288.

[25] G. Wachsmuth, Strong Stationarity for Optimal Control of the Obstacle Problem with Control Constraints. SIAM Journal on Optimization 24 (2014), 1914-1932.

[26] G. Wachsmuth, Mathematical Programs with Complementarity Constraints in Banach Spaces. Journal of Optimization Theory and Applications 166 (2015), 480-507. 
[27] G. Wachsmuth, Strong stationarity for optimization problems with complementarity constraints in absence of polyhedricity. Set-Valued Var. Anal. 25 (2017), 133-175.

[28] G. Wachsmuth, A guided tour of polyhedric sets. Journal of Convex Analysis 26 (2019), 153-188.

[29] I. Yousept, Hyperbolic Maxwell variational inequalities for Bean's critical-state model in type-II superconductivity. SIAM J. Numer. Anal. 55 (2017), 2444-2464.

\section{Acknowledgment}

The research of this work was carried out in Project P16 (Optimal Control of Variational Inequalities of the Second Kind with Application to Yield Stress Fluids) within the DFG Priority Program SPP 1962 (Non-smooth and Complementaritybased Distributed Parameter Systems: Simulation and Hierarchical Optimization). The support by the DFG is gratefully acknowledged.

Constantin Christof

Fakultät für Mathematik, TU München

Boltzmannstraße 3

85748 Garching bei München, Germany

e-mail: christof@ma.tum.de

Christian Meyer

Fakultät für Mathematik, TU Dortmund

Vogelpothsweg 87

44227 Dortmund, Germany

e-mail: cmeyer@math.tu-dortmund.de

Ben Schweizer

Fakultät für Mathematik, TU Dortmund

Vogelpothsweg 87

44227 Dortmund, Germany

e-mail: ben.schweizer@math.tu-dortmund.de

Stefan Turek

Fakultät für Mathematik, TU Dortmund

Vogelpothsweg 87

44227 Dortmund, Germany

e-mail: stefan.turek@math.tu-dortmund.de 